\title{
Atuação do enfermeiro na prevenção da pneumonia associada à ventilação mecânica em pediatria
}

Nurse's role in the prevention of ventilator-associated pneumonia in pediatrics

Papel de la enfermera en la prevención de la neumonía asociada al ventilador en pediatría

Recebido: 07/09/2021 | Revisado: 13/09/2021 | Aceito: 14/09/2021 | Publicado: 16/09/2021

\author{
Ana Fátima Souza Melo de Andrade \\ ORCID: https://orcid.org/0000-0002-7024-6175 \\ Centro Universitário Estácio de Sergipe, Brasil \\ E-mail: anafatimamelo@hotmail.com \\ Weber de Santana Teles \\ ORCID: https://orcid.org/0000-0003-1770-8278 \\ Centro de Hemoterapia de Sergipe, Brasil \\ E-mail: arteecura@hotmail.com \\ Max Cruz da Silva \\ ORCID: https://orcid.org/0000-0002-6944-5986 \\ Faculdade Pio Décimo, Brasil \\ E-mail: maxlfi@hotmail.com \\ Alejandra Debbo \\ ORCID: https://orcid.org/0000-0002-7743-5921 \\ Universidade Tiradentes, Brasil \\ E-mail: aledebbo@hotmail.com \\ Ruth Cristini Torres \\ ORCID: https://orcid.org/0000-0002-8664-192X \\ Instituto de Hematologia e Hemoterapia de Sergipe, Brasil \\ E-mail: ruthcristini@gmail.com \\ Marcel Vinícius Cunha Azevedo \\ ORCID: https://orcid.org/0000-0002-5312-3333 \\ Centro Universitário Estácio Sergipe, Brasil \\ E-mail: marcelvinicius49@gmail.com \\ Taíssa Alice Soledade Calasans \\ ORCID: https://orcid.org/0000-0003-0460-4437 \\ Universidade Tiradentes, Brasil \\ E-mail: taissa.asc@gmail.com \\ Paulo Celso Curvelo Santos Junior \\ ORCID: https://orcid.org/0000-0001-5834-6782 \\ Universidade Tiradentes, Brasil \\ E-mail: paulo.curvelo.jr@gmail.com \\ Ângela Maria Melo Sá Barros \\ ORCID: https://orcid.org/0000-0003-4087-3247 \\ Universidade Federal do Rio de Janeiro, Brasil \\ E-mail: angelsamelo@hotmail.com \\ Maria Hozana Santos Silva \\ ORCID: https://orcid.org/0000-0001-5742-5366 \\ Faculdade Ages de Medicina, Brasil \\ E-mail: hosana_p@hotmail.com
}

\begin{abstract}
Resumo
O presente estudo tem o objetivo de verificar na literatura as evidências científicas sobre o papel do enfermeiro na prevenção da pneumonia associada à ventilação mecânica (PAV), com busca dos artigos na Biblioteca Virtual em Saúde, abrangendo estudos publicados entre 2015 e 2019. A atuação do enfermeiro para o direcionamento da assistência a prevenção de infecção relacionada à ventilação mecânica por meio de intervenções de prevenções de riscos. As bases de dados consultadas para a pesquisa foram: BVS; BDENF; SCIELO. Como critérios de inclusão, foram adotados artigos publicados em português, disponíveis na íntegra entre os anos de 2015 a 2019 . O estudo evidenciou a PAV pode trazer grave repercussão para o paciente acometido por essa condição e apresenta um grande impacto nas taxas de morbimortalidade, no tempo de VM, no tempo de permanência na UTI. Diante disso, é fundamental a seleção e a aplicação de medidas baseadas em evidências, apropriadas para cada serviço de saúde e de acordo com as necessidades individuais dos pacientes, visto que tais medidas, comprovadamente, quando aplicadas coletivamente, diminuem a densidade de incidência de PAV, uma das mais frequentes infecções relacionadas à
\end{abstract}


assistência à saúde nas UTIs, nesse contexto o enfermeiro desempenha um papel fundamental do cuidar, junto à equipe assistencial, na prevenção de infecção, devendo estar capacitado a exercer as atividades de maior complexidade, respaldado em conhecimentos científicos concretizados a partir da prática cotidiana de cuidar e da pesquisa, a fim de conduzir um atendimento com autoconfiança e segurança.

Palavras-chave: Respiração artificial; Cuidados de Enfermagem; Pneumonia; Pediatria.

\begin{abstract}
This study aims to verify in the literature the scientific evidence on the role of nurses in the prevention of ventilatorassociated pneumonia (VAP), with a search for articles in the Virtual Health Library, covering studies published between 2015 and 2019. of nurses to direct assistance to the prevention of infection related to mechanical ventilation through risk prevention interventions. The databases consulted for the research were: VHL; BDENF; SCIELO. As inclusion criteria, articles published in Portuguese were adopted, available in full between the years 2015 to 2019 . The study showed that VAP can have serious repercussions for the patient affected by this condition and has a great impact on morbidity and mortality rates, in the time on MV, in the length of stay in the ICU. Therefore, it is essential to select and apply evidence-based measures, appropriate for each health service and according to the individual needs of patients, since such measures, when applied collectively, have been proven to reduce the incidence density of VAP , one of the most frequent infections related to health care in ICUs, in this context, nurses play a fundamental role in care, together with the care team, in the prevention of infection, and must be able to perform the most complex activities, supported by scientific knowledge. implemented from the daily practice of caring and research, in order to conduct care with self-confidence and security.
\end{abstract}

Keywords: Artificial respiration; Nursing care; Pneumonia; Pediatrics.

\title{
Resumen
}

Este estudio tiene como objetivo verificar en la literatura la evidencia científica sobre el papel de las enfermeras en la prevención de la neumonía asociada al ventilador (NAV), con una búsqueda de artículos en la Biblioteca Virtual en Salud, cubriendo estudios publicados entre 2015 y 2019. Asistencia directa a la prevención de infecciones relacionadas con la ventilación mecánica mediante intervenciones de prevención de riesgos. Las bases de datos consultadas para la investigación fueron: BVS; BDENF; SCIELO. Como criterio de inclusión se adoptaron artículos publicados en portugués, disponibles íntegramente entre los años 2015 a 2019. El estudio mostró que la NAV puede tener graves repercusiones para el paciente afectado por esta condición y tiene un gran impacto en las tasas de morbimortalidad, en el tiempo en VM, en el tiempo de estancia en UCI. Por tanto, es fundamental seleccionar y aplicar medidas basadas en la evidencia, adecuadas para cada servicio de salud y de acuerdo con las necesidades individuales de los pacientes, ya que dichas medidas, aplicadas de forma colectiva, han demostrado reducir la densidad de incidencia de NAV, una de las más importantes. infecciones más frecuentes relacionadas con la atención de la salud en las UCI, en este contexto, el enfermero juega un papel fundamental en la atención, junto con el equipo de atención, en la prevención de la infección, y debe ser capaz de realizar las actividades más complejas, apoyado en el conocimiento científico. implementado desde la práctica diaria del cuidado y la investigación, con el fin de realizar el cuidado con confianza y seguridad.

Palabras clave: Respiración artificial; Cuidado de enfermera; Neumonía; Pediatría.

\section{Introdução}

A pneumonia é um processo inflamatório pulmonar, podendo ser ocasionada por diversos patógenos, incluindo bactérias, micobactérias, fungos e vírus. As pneumonias podem ser divididas em quatro tipos: pneumonia adquirida na comunidade (PAC), pneumonia associada a cuidados de saúde (PACS), pneumonia adquirida no hospital (PAH) e pneumonia associada à ventilação mecânica (PAV) (Mansano et al., 2017).

As técnicas modernas e complexas utilizadas nas Unidades de Terapia Intensiva (UTI) aumentam a chance de sobrevivência dos clientes gravemente enfermos, no entanto os mesmos são expostos a riscos e ficam predispostos a contraírem Infecções Relacionadas à Assistência à Saúde (IRAS). Dentre outros riscos, pode-se citar os métodos invasivos que o cliente é submetido durante a internação em UTI, como cateterismos venosos e vesicais e a ventilação mecânica (Mota et al., 2017).

Ventilação mecânica trata-se da utilização de um equipamento que atua na substituição total ou parcial da atividade ventilatória do cliente, tendo como objetivo manter o equilíbrio entre oferta e demanda de oxigênio aos clientes que estejam impossibilitados de manterem suas funções respiratórias (Figura 1). 
Figura 1 - Ventilador pulmonar mecânico.

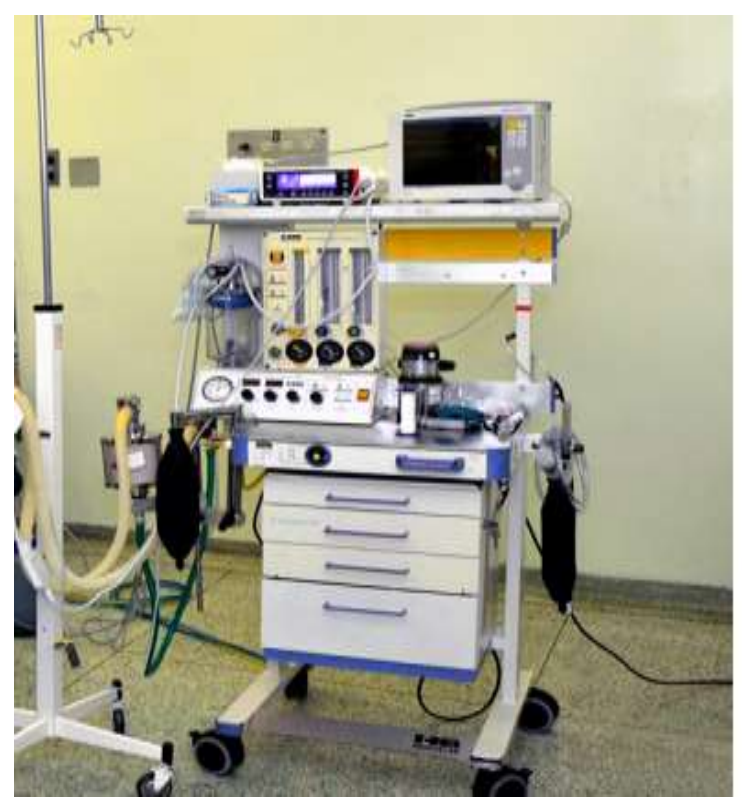

Fonte: Jornal da Unicamp Campinas, (2013), Nº 574.

É uma técnica utilizada em grande escala em UTI, atualmente, a maior parte dos ventiladores artificiais contém telas nas quais se podem observar as curvas de volume, fluxo e pressão ao longo do tempo, como demonstra a Figura 2.

Figura 2 - Curva de Fluxo - Ventilação controlada por volume.

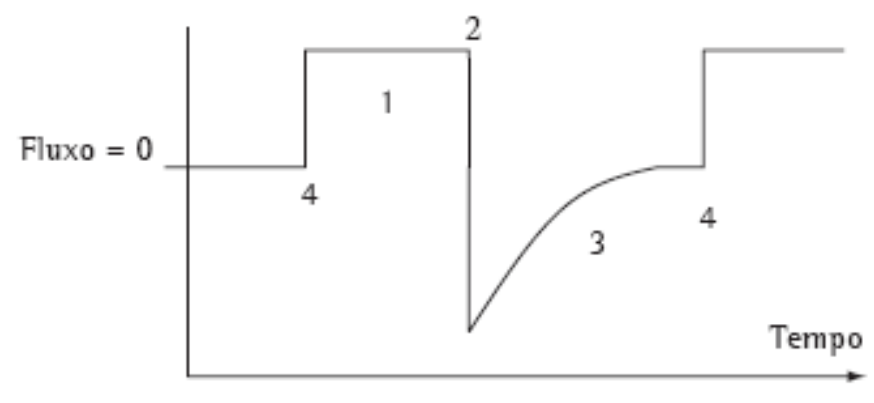

Fonte: Carvalho et al. (2017).

$\mathrm{Na}$ fase 1, o ventilador efetua a insuflação no pulmão, na fase 2 mudança da fase inspiratória para expiratória, na fase 3, fechação da válvula inspiratória e abertura da válvula expiratória, na fase 4 , modificação da etapa expiratória para a inspiratória (Carvalho et al., 2017).

A pneumonia que se manifesta durante o período de 48 horas após a intubação ou 72 horas após a extubação, a que foi descartada a possibilidade de estar incubada durante a admissão do cliente é definida como Pneumonia Associada à Ventilação Mecânica (PAV). Tal complicação possui fatores de risco como idade acima de 70 anos, diminuição do nível de consciência, intubação e reintubação traqueal, situação imunológica, utilização de imunossupressores, choque, Doença Pulmonar Obstrutiva Crônica (DPOC), tempo de ventilação invasiva maior que sete dias, aspiração de conteúdo contaminado presente no circuito do respirador mecânico, desnutrição, infecção exógena, uso de antimicrobianos profilaticamente e pH gástrico > 4 (Rodrigues et al., 2016). 
A higienização das mãos é dada como a técnica isolada que mais tem o poder de controlar as IRAS, no entanto, ocorre grande falta de adesão a essa prática por parte dos profissionais de saúde. Apenas com o uso de água e sabão durante a higienização das mãos, pode-se reduzir a população microbiana presente nas mãos e, muitas vezes, bloquear a cadeia de propagação de doenças (Araújo et al., 2016).

A prevenção da PAV tem como finalidade diminuir a instalação de reservatórios de microrganismos potenciais, prevenir a inserção destes reservatórios nos alvéolos pulmonares e contribuir para a proteção do cliente submetido a procedimentos invasivos. Esta ação pode reduzir o uso de medicamentos antimicrobianos, o tempo de internação e a mortalidade relacionada à PAV (Moreira et al., 2011).

O enfermeiro tem atuação importante nas práticas que envolvem ventilação mecânica invasiva e não invasiva, na sistematização da assistência de enfermagem desde a instauração, manipulação, precaução e controle de eventos adversos, em medidas de contenção de infecção até cuidados com o equipamento. A assistência do enfermeiro ao cliente em ventilação mecânica torna-se intensa e complexa, tendo este profissional uma grande importância na recuperação da saúde do mesmo (Amorim \& Gomes, 2015).

Diante dessa temática, objetiva-se, através deste estudo, verificar na literatura as evidências científicas sobre o papel do enfermeiro na prevenção de PAV.

\section{Metodologia}

Trata-se de um estudo de revisão integrativa da literatura, de caráter exploratório, descritivo e documental, abrangendo o período de 2015 a 2019.

As bases de dados consultadas para a pesquisa foram: BVS (Biblioteca Virtual em Saúde); BDENF (Base de Dados Bibliográfica Especializada na Área de Enfermagem); SCIELO (Biblioteca Científica Eletrônica Virtual). Como critérios de inclusão, foram adotados artigos publicados em português, disponíveis na íntegra entre os anos de 2015 a 2019 e como critérios de exclusão, artigos pagos para seu acesso e que não responderam aos objetivos do projeto.

A amostra inicial do estudo foi composta por 30 artigos. Destes, foram excluídos 15 por não atenderem aos critérios de inclusão. Os 15 artigos restantes foram todos utilizados na íntegra.

A coleta de dados foi realizada em duas etapas: na primeira foram estabelecidos os descritores mediante busca nos Descritores em Ciências da Saúde - DECS, disponível em http://decs.bvs.br. Sendo assim, foram estabelecidos: enfermeiro, Ventilação Mecânica; Atuação do enfermeiro; Pneumonia. Para pesquisa nos bancos de dados, estes foram utilizados individualmente e agregados (Foram utilizados os descritores específicos do tema proposto: Respiração artificial; Cuidados de Enfermagem; Pneumonia; Pediatria. Estes foram cruzados por meio dos operadores booleanos "AND" e "ON “, formando as seguintes estratégias de busca: enfermeiro AND ventilação mecânica; atuação do enfermeiro AND pneumonia OR.

Para análise de dados foi utilizada a avaliação qualitativa, por visar à compreensão interpretativa dos fatos, colocando o pesquisador diante de um material de trabalho constituído que o levará a trilhar por caminhos subjetivos em busca da complexidade dos fenômenos e da sua compreensão que só ocorrerá se a ação for colocada dentro de um conjunto de significados/categorização (Goldenberg, 2010).

\section{Resultados e Discussão}

\subsection{Atuação do enfermeiro frente à prevenção de pneumonia associada à ventilação mecânica (PAV) na uti pediatrica}

A criação e implementação de protocolos dentro das UTIs é citada como uma estratégia eficaz na prevenção de PAV, quando aplicados de forma multidisciplinar e auditados pelos Serviços de Controle de Infecção Hospitalar. O trabalho do 
enfermeiro na UTI é um desafio, pois requer responsabilidade, vigilância e respeito, o paciente não pode externar suas necessidades, é demasiadamente vulnerável e isso o torna totalmente dependente da equipe que está lhe dando assistência.

Os enfermeiros ocupam uma posição singular na execução dos componentes dos bundles de prevenção de PAV e que, para haver sucesso na aplicação destes pacotes, os mesmos devem ser implementados de forma coordenada e com liderança, a aspiração de secreções subglóticas e o uso de clorexidina para realização de higiene oral, pausa na sedação e pausa diária na administração de relaxante muscular são ações menos executadas por enfermeiros (Hill, 2016).

É necessário que se estabeleça uma relação tanto entre o cuidador e o ser cuidado no atendimento da Enfermagem nas ações de prevenção de ocorrência da pneumonia associada à ventilação mecânica, utilizando-se de conhecimento, respaldadas na ciência, com um olhar subjetivo, envolvendo respeito e empatia.

O cuidado em UTI se tornou uma das áreas da enfermagem em constante desenvolvimento, exigindo desses profissionais a busca contínua por atualização e capacitação, utilizando-se do avanço tecnológico, que se tornou uma ferramenta muito importante para a sobrevivência do bebê. Conhecer as medidas de prevenção às infecções proporciona ao enfermeiro melhores condições de gestão para a tomada de decisões.

\subsection{Fatores de risco e métodos preventivos}

A prevenção da PAV tem por objetivo garantir a diminuição da proliferação e transmissão de patógenos ao paciente que está em ventilação mecânica (VM), reduzindo a colonização dos reservatórios por patógenos potenciais; prevenindo a inoculação para os alvéolos pulmonares; e auxiliar nas defesas do doente crítico internado na UTI.

A atuação do enfermeiro dentro da UTI é uma ação de grande e constante desafio, pois para que suas ações sejam efetivas, é necessário que o profissional tenha características específicas, como habilidade, constante vigilância, além de muito respeito, pois o paciente em questão é demasiadamente sensível e vulnerável, tornando-o totalmente dependente da equipe que o está assistindo.

O planejamento de cuidados de enfermagem para estes pacientes que, geralmente são gravemente enfermos, depende de um processo extremamente rigoroso, baseado em uma criteriosa avaliação para determinar a eficácia deste planejamento.

Em UTI, a rotina de trabalho cansativa e excessiva e a falta de apoio de recursos e profissionais no âmbito hospitalar interferem diretamente na linha de cuidado. A enfermagem tecnicista e a mecanização do trabalho levam o profissional a deixar de lado as preconizações ao paciente e agir apenas de maneira técnica. Olhando apenas a doença e não o indivíduo como um todo, observamos que os profissionais diretamente ligados aos cuidados dos recém-nascidos na UTI não estão completamente orientados nas medidas preventivas, especificamente da Pneumonia Associada Ventilação Mecânica. A falta de treinamento destes profissionais pode resultar no favorecimento na elevação dos riscos de infecções.

\section{Considerações Finais}

O enfermeiro desempenha um papel fundamental, junto à equipe assistencial, na prevenção de PAV, porém, ele deve estar capacitado a exercer as atividades de maior complexidade, respaldado em conhecimentos científicos concretizados a partir da prática cotidiana de cuidar e da pesquisa, a fim de conduzir um atendimento com autoconfiança e segurança.

Ao enfermeiro, por ser o líder da equipe de enfermagem, cabe disseminar conhecimentos sobre práticas de prevenção de PAV, bem como instituir medidas cientificamente comprovadas para prevenção dessa infecção. Os custos com tratamento de saúde são menores quando se instituem medidas de prevenção de PAV. O gasto com compra de insumos se torna positivo frente às complicações e gastos possíveis, caso o cliente adquira PAV. 
A sistematização da assistência de enfermagem é de suma importância no contexto de prevenção de PAV, pois através dela o enfermeiro poderá avaliar as condições do cliente e tomar decisões cruciais, o que confere maior segurança para o cliente e para o profissional e consequentemente melhora a qualidade da assistência prestada. Os cuidados com a manipulação dos dispositivos e os protocolos institucionais são barreiras de infeções que uma assistência com segurança evita infeções e danos ao paciente, evitando a morte.

Desta forma, torna-se indispensável a realização de novos trabalhos que abordem condutas de profissionais da saúde quanto aos cuidados com pacientes em tratamento com ventilação mecânica.

\section{Referências}

Amorim, M .M., \& Gomes, S.R. (2015). Ações de Enfermagem para Prevenção de Infecções Associadas à Ventilação Mecânica na Unidade de Terapia Intensiva Neonatal. Revista Interdisciplinar do Pensamento Científico, Rio de Janeiro. 1(2), 72-82.

Araújo, D. D. (2016). A Importância da Higienização das Mãos no Controle das Infecções em Serviços de Saúde. Revista de Enfermagem UFPE Online. Recife. 10(6), $4080-84$.

Bardin, L. (2011). Análise de conteúdo. São Paulo: Edições 70.

Brasil. Ministério da Saúde (2009). Infecções do trato respiratório -orientações para prevenção de infecções relacionadas à assistência à saúde. Brasília.

Brasil. Ministério da Saúde (2017). Medidas de Prevenção de Infecção Relacionada à Assistência à Saúde. Brasília.

Carvalho, C. R. R., Junior, C. T., \& Franca, S. A. (2007). Ventilação mecânica: princípios, análise gráfica e modalidades ventilatórias. J. bras. pneumol. 33(2).

Gomes, A. M., \& Silva, R. C. L. (2010). Bundle de prevenção da pneumonia associada à ventilação mecânica: o que sabem os enfermeiros a esse respeito?. Revista Cuidado é Fundamental Online. Rio de Janeiro. 2, 562-567.

Gonçalves, F. A. F. (2012). Eficácia de estratégias educativas para ações preventivas da pneumonia associada à ventilação mecânica. Escola Anna Nery, Rio de Janeiro. 16(4), 802-808.

Halpin, L. (2013). Ventilator-Associated Pneumonia Among Cardiac Surgery Patients What Can We Do for Prevention?. Journal of Nursing Care Quality, Virginia. 28(4), 345-351.

Hill, C. (2016). Nurse-led implementation of a ventilator-associated pneumonia care bundle in a children's critical care unit. Royal College of Nursing, London. 28(4), 23-27.

Gardenal, I. (2013). Ondas sonoras desobstruem vias respiratórias, Jornal da UNICAMP, 574.

Kiyoshi, H., \& Blegen, M. (2015). Influence of Institutional Guidelines on Oral Hygiene Practices in Intensive Care Units. American Journal of Critical Care, Columbia. 24(4), 309-17.

Mendes, K. D. S., Silveira, R .C. C. P., \& Galvão, C. M. (2008). Revisão Integrativa: Método de Pesquisa para a Incorporação de Evidências na Saúde e na Enfermagem.Texto \& Contexto Enfermagem. 17(4).

Micik, S. (2013). Reducing risk for ventilator associated pneumonia through nursing sensitive interventions. Intensive and Critical Care Nurse, Phoenix. $29(5), 261-65$.

Moher, D. (2009). Reprint Preferred Reporting Items for Systematic Reviews and Meta- Analyses: The PRISMA Statement. Physical Therapy, Oxford. 89(9), 873-80.

Moreira, B. S. G. (2011). Pneumonia associada à ventilação mecânica: medidas preventivas conhecidas pelo enfermeiro. Revista Baiana de Enfermagem. $25(2)$.

Mota, E. C., et al. (2017). Incidência de Pneumonia Associada à Ventilação Mecânica em unidade de terapia intensiva. Revista de Medicina da USP, São Paulo. 50(1), 39-46

Munro, C. L. (2014). Oral Health: Something to Smile About! American Journal of Critical Care, Columbia. 23(4), 282-88.

Mansano, F., Belei, R. A., Vinci, L. A. S., Melo, B. L. D., Cardoso, L. T. Q., Garcia, J. C. P., \& Carrilho, C. M. D .M. (2017). Impacto de ação educativa na manutenção do decúbito elevado como medida preventiva de pneumonia associada à ventilação mecânica em Unidade de Terapia Intensiva. Abcs Health Sciences. 42(1), 21-6.

Prendergast, V., Kleiman, C., \& King, M. (2013). The Bedside Oral Exam and the barrow Oral Care Protocol: Translating evidence-based oral care into practice. Intensive and Critical Care Nurse, Phoenix. 29(5), 282-290.

Rodrigues, A. N., et al. (2016). Impactos e fatores determinantes no bundle de pneumonia associada à ventilação mecânica. Revista Brasileira de Enfermagem. Brasília. 69(6). 
Research, Society and Development, v. 10, n. 12, e156101220328, 2021

(CC BY 4.0) | ISSN 2525-3409 | DOI: http://dx.doi.org/10.33448/rsd-v10i12.20328

Shimabukuro, P. M. S., Paulon, P., \& Feldman, L. B. (2014). Implantação de bundles em unidade de terapia intensiva: um relato de experiência. Revista de Enfermagem da UFSM, Santa Maria. 4(1), 227-236.

Silva, S. G., et al. (2014). Avaliação de um bundle de prevenção da pneumonia associada à ventilação mecânica em unidade de terapia intensiva. Escola Anna Nery, Rio de Janeiro. 18(2), 290-295.

Silva, M. C. O., \& Moura, R. C. M. (2016). Cuidados de enfermagem na prevenção da pneumonia associada à ventilação mecânica: revisão integrativa. Revista Cultural e Científica do UNIFACEX, Natal. 14(2), 74-85.

Soares, C. B., et al. (2014). Revisão integrativa: conceitos e métodos utilizados na enfermagem. Revista da Escola de Enfermagem da USP, São Paulo. 48(2), 335-345. 